\title{
DISCRIMINATION OF HOMOLOGOUS CHROMOSOMES OF MAIZE WITH GIEMSA STAINING
}

\author{
GY. HADLACZKY \\ Institute of Genetics, Biological Research Centre, Szeged, Hungary \\ and \\ L. KÁLMÁN \\ Cereal Research Institute, Szeged, Hungary
}

Received 2.vi.75

\section{SUMMARY}

\begin{abstract}
A Giemsa method is described for the identification of somatic chromosomes of maize (Zea mays L.). Three inbred stocks and their hybrids were examined. The Giemsa method proved to be useful for the characterisation of different stocks. On the basis of the different staining properties of homologous chromosomes, the parental and grandparental chromosomes were identified in the hybrids.
\end{abstract}

\section{INTRODUGTION}

MAIZE is one of the most important cultivated cereals of the world and it is the best known cytologically studied crop so far. Kuwada (1911) reported the exact chromosome number of maize $(2 n=20)$ and McClintock (1929) described the morphological characteristics of the chromosomes on the basis of their relative lengths, arm ratios and the positions of knobs at the pachytene stage of meiosis. Creighton and McClintock (1931) using the knobs as cytological markers for the identification of individual chromosomes, demonstrated the correlation between cytological and genetic crossing-over. Longley (1939) described 22 different positions of knobs and showed that knob positions are suitable for the discrimination of stocks. He found variable numbers of knobs in different stocks and in some no knobs at all. From different studies Neuffer, Jones and Zuber (1968) gave an account of the distribution of knobs on maize chromosomes.

Identification of the somatic chromosomes of maize is difficult because they are relatively small and the knob structures are invisible by conventional chromosome staining. Lately, the somatic karyotype of maize has been described by Chen (1969) and Filion and Walden (1973) on the basis of relative lengths, arm ratios and presence of satellite.

Giemsa staining is a very useful cytological tool for detection of heterochromatin in plant chromosomes and nuclei. The denaturation-reannealing method which has recently been devised by Vosa and Marchi (1972) produces characteristic banding patterns on somatic metaphase chromosomes of plants. Sartori and Ting (1974), with a modified Sumner's technique, showed prominent heterochromatic bands on the chromosomes 6,7 and 9 in haploid maize.

In the present study an attempt was made to characterise the somatic 
chromosomes in diploid stocks of maize, using a modified method of Vosa and Marchi (Hadlaczky and Koczka, 1974; Hadlaczky and Belea, 1975).

\section{Materials AND Methods}

The material consisted of seeds of three inbred stocks viz. PEM, GK2, GK 4 and two of their hybrids GK2 $\times$ GK 4 and $(\mathrm{GK} 2 \times \mathrm{GK} 4) \times \mathrm{PEM}$. The PEM stock (Purple Embryo Marker: $b$ pl $A C R^{n j}$ Cudu pr $D^{w_{5}}$ ) which was produced by Dr S. S. Ghase has been kindly provided by All-Union Research Institute of Plant Industry, Leningrad; U.S.S.R. GK2 and GK4 (sublines of the American 153R and G103 stocks, respectively) and the hybrid stocks were produced by the Hungarian Cereal Research Institute, Szeged. In addition, the stocks WF9, B14, GK15 and A619 were used for comparing the heterochromatic staining of nuclei.

The seeds were germinated on wet filter paper in petri dishes for about 4 days. Actively growing primary root-tips were pretreated with a solution of 0.5 per cent monobromonaphthalene and 0.05 per cent colchicine at room temperature for 4 hours and fixed in 1:3 acetic alcohol for 1-12 hours. The root-tips were softened by a solution of 2 per cent cellulase (Onozuka R-10) and 2 per cent pectinase (Fluka) for 5-8 hours at room temperature and then squashed in 45 per cent acetic acid. The slides were frozen on dry ice or liquid nitrogen and, after removing the coverslips, they were transferred to absolute alcohol and air dried. The preparations were immersed in a saturated solution of barium hydroxide for 30-35 minutes at $60^{\circ} \mathrm{C}$. After washing with distilled water they were incubated in $2 \times \mathrm{SSG}$ at $60^{\circ} \mathrm{C}$ for 60 minutes at $p \mathrm{H} 7 \cdot 0$. Giemsa stain was freshly prepared from Gurr's stock solution by diluting it $50 \times$ with Sörensen phosphate buffer at $p \mathrm{H} 6.8$. Stained slides were washed with distilled water, air dried, immersed in xylene and mounted in Gurr's DPX.

\section{Results}

A Giemsa stained metaphase plate of PEM stock is presented in fig. 1. Five pairs of the chromosome complement show dark and distinct heterochromatic bands and two pairs have small terminal knobs. Using the heterochromatic banding patterns as well as the arm ratios and relative lengths, the chromosomes can be identified as follows:

The chromosomes 1, 6, 7, 8, 9 and 10 show thin centromeric heterochromatic knobs whereas in the chromosomes 2, 3, 4 and 5 no heterochromatic staining was found at the centromeric region.

Chromosome 1. The short arm has a heterochromatic knob at the distal portion.

Chromosome 2. A very intensive heterochromatic region is present near to the telomeric end of the long arm.

Chromosome 3. A small terminal knob exists on the long arm.

Chromosomes 4 and 5. These chromosomes appear to have no heterochromatin.

Chromosome 6. The short arm possesses an intensive heterochromatic satellite and a thick heterochromatic band. The long arm has an interstitial band in the distal third. 
Plate 1

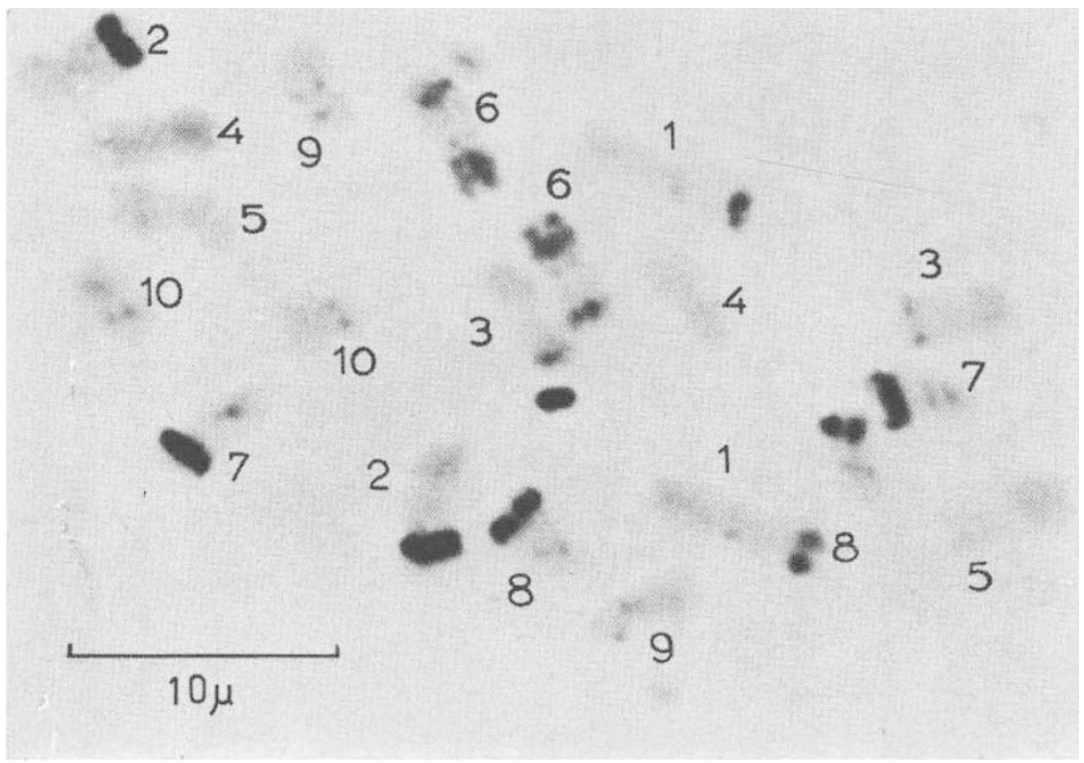

Fic. 1.-Giemsa stained somatic metaphase from the PEM stock of maize.

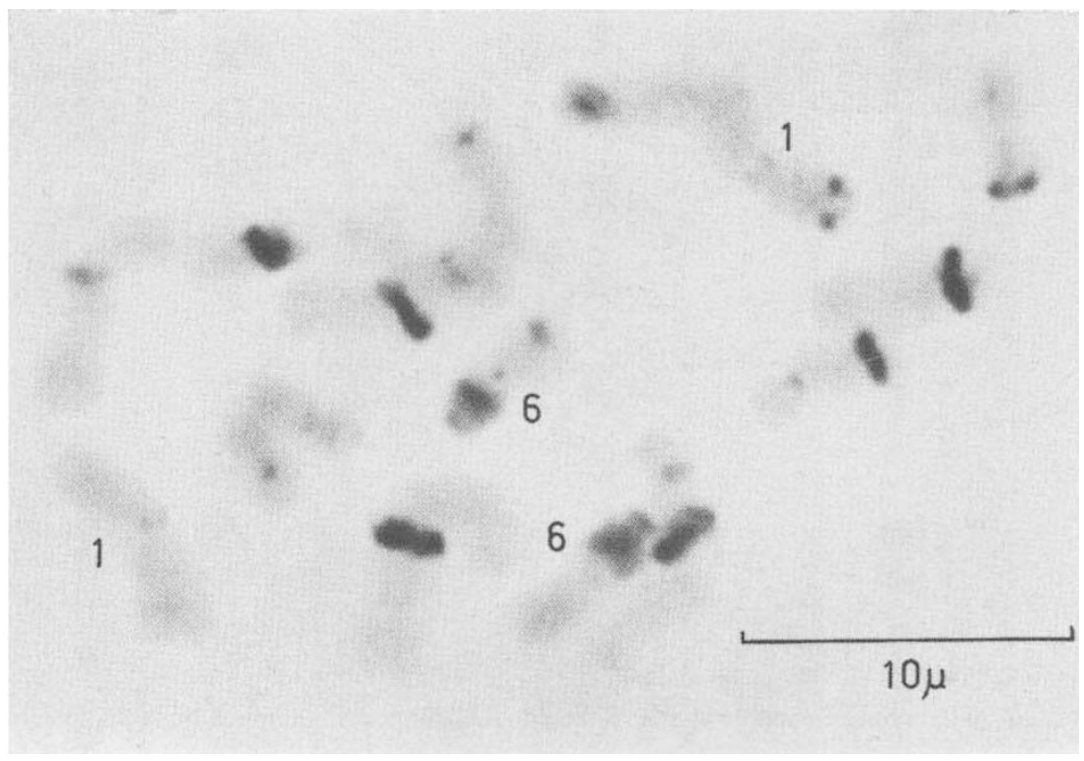

FIG. 2.- - Somatic metaphase from the $(\mathrm{GK} 2 \times \mathrm{GK} 4) \times \mathrm{PEM}$ hybrid. Note the different staining characteristics of homologous chromosomes. 


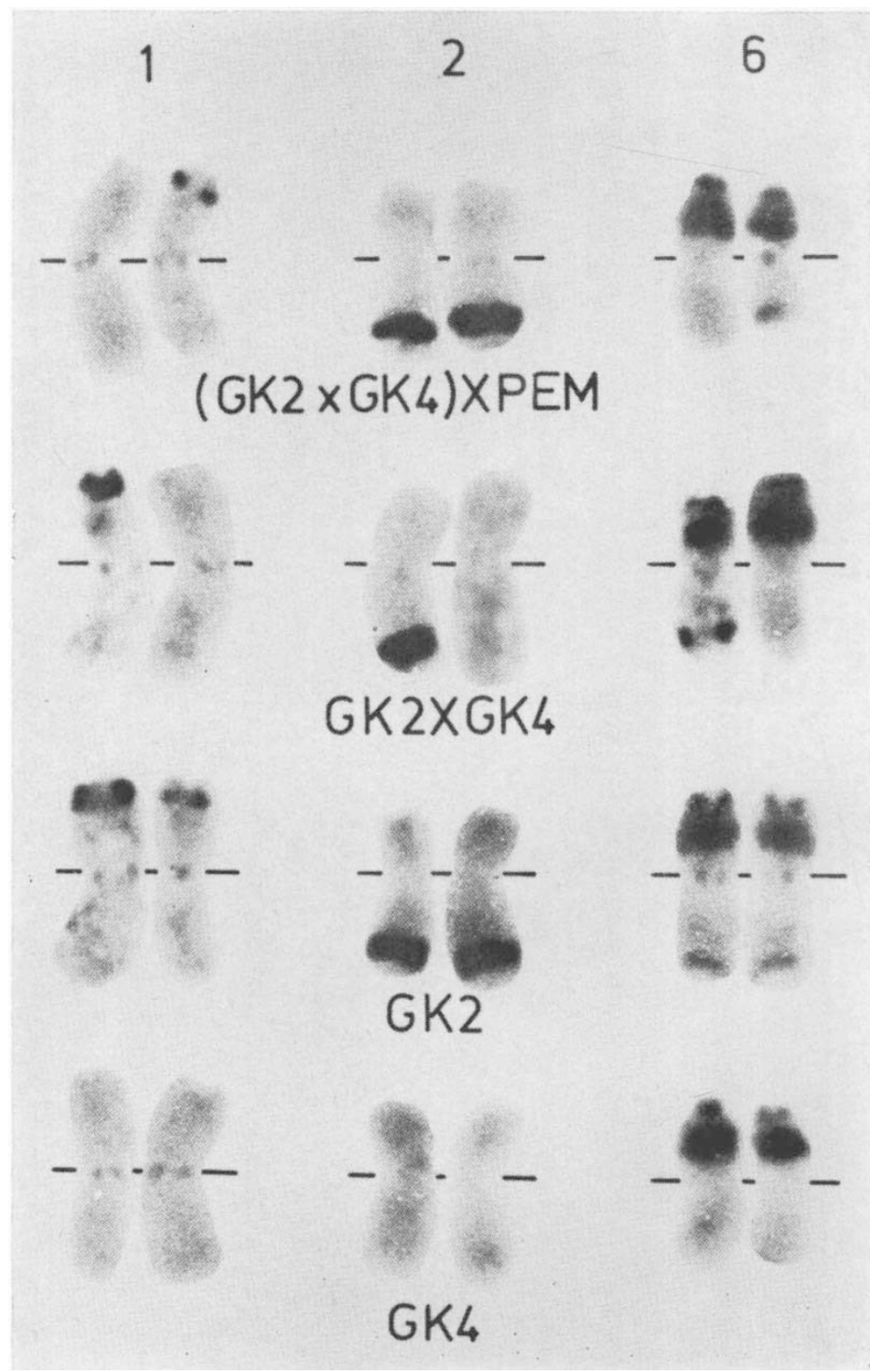

FIG. 3.-Differential staining patterns of the 1,2 and 6 chromosomes in the (GK2 $\times$ GK4) $\times$ PEM hybrid and in the parental stocks after Giemsa staining. 


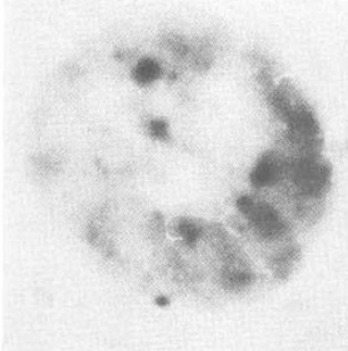

GK4

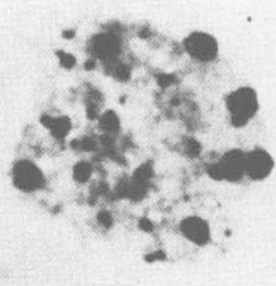

GK2

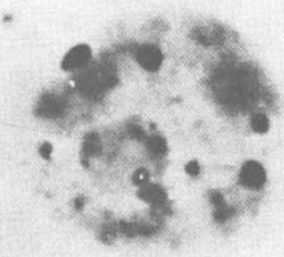

GK2 2 GK4

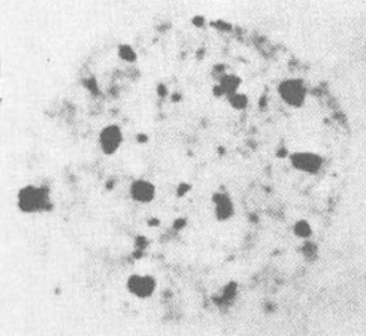

PEM

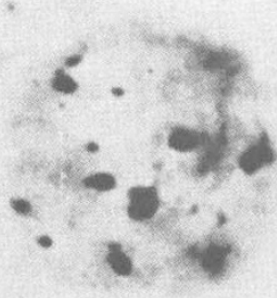

$(G K 2 \times G K 4) \times P E M$

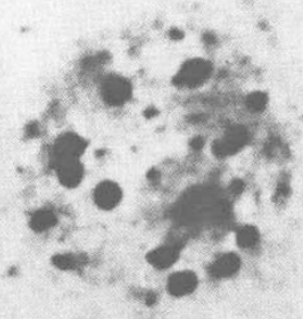

WF9

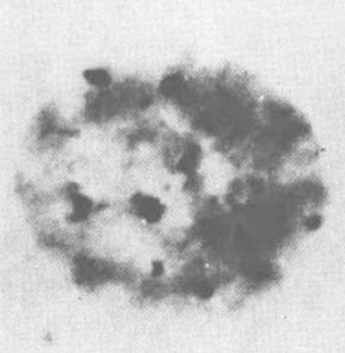

B14

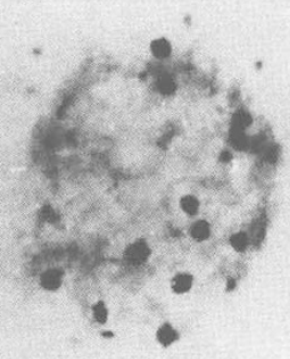

GK15

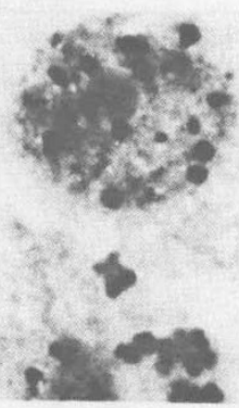

A 619

FIG. 4.- Heterochromatin at interphase from different stocks and hybrids of maize. 
Chromosome 7. A deeply stained heterochromatic band is present near to the telomeric end of the long arm.

Chromosome 8. The long arm has a terminal band of more or less the same intensity as that of chromosome 7.

Chromosome 9. A small heterochromatic knob is located on the telomeric end of the short arm.

Chromosome 10. No heterochromatic knob or band is present except the centromeric heterochromatin.

Figure 2 shows the differential Giemsa staining of homologous chromosomes of the (GK2 $\times$ GK4) $\times$ PEM hybrid. It is especially conspicuous in the cases of chromosomes 1 and 6; one of the chromosomes of the first homologous pair is distinguishable from the other by the presence of a heterochromatic knob at the distal end of the short arm, while the chromosomes of the sixth homologous pair differ from each other with respect to heterochromatin on the long arm and at the centromere.

The clear-cut differences in the heterochromatic pattern facilitates the characterisation of chromosome complements of different stocks and hybrids. The detection of parental homologous chromosomes of GK2 $\times$ GK 4 hybrids is very easy because the heterochromatin has been observed in six chromosome pairs of the GK2 stock but only in the sixth and ninth pairs of GK4 stock. The three easily identifiable chromosome pairs (1, 2 and 6) are chosen for comparisons of hybrids with parents (fig. 3 ).

The origin of the knobless chromosomes of the GK $2 \times \mathrm{GK} 4$ hybrid can be traced back to one of its parents, GK4. The knobless chromosomes of first and sixth pairs in (GK2 $\times$ GK4) $\times$ PEM hybrid have come from GK4 stock and one of the chromosomes of the second pair from GK2 stock.

Differences in the heterochromatic staining patterns could also be found in interphase nuclei (fig. 4). The interphase nuclei of hybrids were observed to have an intermediate number of heterochromatic regions to that found in nuclei of parents. The largest differences in heterochromatic staining characteristics were found between the GK4 and WF9 stocks where the number of cells analysed was 32 and 35 respectively. The average value of large heterochromatic knobs in GK4 nuclei was 2.0 whereas in nuclei of WF9 was 15.9. In the nucleus of GK4 stock, the two large knobs correspond to the heterochromatic regions of chromosome 6 .

The different stainability of the centromeric and extra-centromeric heterochromatin reveals that these two types of heterochromatin are not the same. Similar findings were described by Vosa (1974) and Hadlaczky and Koczka (1974) in rye chromosomes.

From the relation of the localisation of the heterochromatic bands to the distribution of knobs on pachytene chromosomes it appears that the Giemsa procedure is a comparatively simple method to detect knobs on somatic chromosomes of different stocks and hybrids of maize. The heterochromatic banding pattern and its correspondence with the knobs on pachytene chromosomes may be useful in maize cytogenetics such as chromosome mapping, detection of hybrid seeds and tracing out the ancestry of different stocks.

Acknowledgment.-The authors wish to thank Mr L. V. Reddy for his help in the preparation of manuscript. 


\section{References}

CHEN, c. c. 1969. The somatic chromosomes of maize. Can. 7. Genet. Cytol., 11, 752-754.

CREIGHTON, H. B., AND MCCLINTOCK, B. 1931. A correlation of cytological and genetical crossing-over in Zea mays. Proc. Nat. Acad. Sci., U.S.A., 17, 492-497.

FILION, W. G., AND WALDEN, D. B. 1973. Karyotype analysis: The detection of chromosomal alterations in the somatic karyotype of Zea mays $\mathrm{L}$. Chromosoma, Berl., 41, 183-194.

HADLACZKY, GY., AND KOCZKA, K. 1974. C-banding karyotype of rye from hexaploid Triticale. Cereal Res. Communications, 2, 193-200.

HADLACZKY, GY., AND BELEA, A. 1975. C-banding in wheat evolutionary cytogenetics. Plant Sci. Letters, 4, 85-88.

KuWADA, x. 1911. Meiosis in the pollen mother cells of Zea mays L. Bot. Mag. (Tokyo), 25, 163-181.

longley, A. E. 1939. Knob position on corn chromosomes. F. Agr. Res., 59, 475-490.

mcclintock, B. 1929. Chromosome morphology in Zea mays. Science, 69, 629.

NEUfFer, M. G., JONES, L., AND ZUBER, M. s. 1968. The mutants of maize. Crop Science Society of America, Madison, Wisconsin.

SARTORI, L., AND TING, Y. c. 1974. Giemsa banding in the chromosomes of haploid maize. Amer. F. Bot. (Suppl.), 61, 63.

VOSA, C. G., AND MARCHI, P. 1972. Quinacrine fluorescence and Giemsa staining in plants. Nature, New Biol., 237, 191-192.

$\operatorname{vosA}$, c. c. 1974. The basic karyotype of rye (Secale cereale) analysed with Giemsa and fluorescence method. Heredity, 33, 403-408. 